\title{
Erratum: On Newton-Cartan trace anomalies
}

\author{
Roberto Auzzi, ${ }^{a, b}$ Stefano Baiguera ${ }^{a}$ and Giuseppe Nardelli ${ }^{a, c}$ \\ ${ }^{a}$ Dipartimento di Matematica e Fisica, Università Cattolica del Sacro Cuore, \\ Via Musei 41, 25121 Brescia, Italy \\ ${ }^{b}$ INFN Sezione di Perugia, \\ Via A. Pascoli, 06123 Perugia, Italy \\ ${ }^{c}$ TIFPA - INFN, c/o Dipartimento di Fisica, Università di Trento, \\ 38123 Povo (TN), Italy \\ E-mail: roberto.auzzi@unicatt.it, giuseppe.nardelli@unicatt.it, \\ stefano.baiguera92@gmail.com
}

ERRATUM TO: JHEP02(2016)003

ArXiv EPrint: 1511.08150 
Due to an overcounting of the number of linearly-independent terms in the basis, the type A anomaly disappears because it can be eliminated by local counterterms $\mathcal{A}_{\mathrm{ct}}$. The complete anomaly then is:

$$
2 T_{0}^{0}+T_{i}^{i}=\mathcal{A}=b \sigma J^{2}+\mathcal{A}_{\mathrm{ct}} .
$$

This is in agreement with [1].

The basis of the anomaly in eq. (3.18) turns out to be redundant, due to the presence of the following relations (which are valid if the Frobenius condition $n \wedge d n=0$ holds):

$$
\begin{aligned}
W^{2} & =12 J^{2}, \\
E_{4} & =72 \chi^{2}-4 \chi R-48 \chi \Omega+8 \Omega^{2}-8 \Omega_{A B} \Omega^{A B}, \\
\left(R_{A B}+2 \Omega_{A B}\right) w^{A} w^{B} & =8 \chi(R-6 \chi+4 \Omega), \\
\left(R_{A B}+2 \Omega_{A B}\right) \Omega^{A B} & =12 \chi^{2}+\frac{1}{2} \Omega(R+4 \Omega)-\chi(R+9 \Omega) .
\end{aligned}
$$

Consequently, the elements $A_{1}, A_{2}, A_{11}$ and $A_{13}$ can be written as linear combinations of the remaining terms. The basis of commutators of two Weyl variations in eq. (3.23) is also redundant:

$$
C_{8}=8 C_{2}-4 C_{3}+\frac{1}{2} C_{4}-5 C_{5}+2 C_{6} .
$$

Then, the matrix in eq. (3.24) becomes

$$
\left(M^{t}\right)^{m k}=\left(\begin{array}{cccccccccccc}
0 & 12 & 0 & 0 & 0 & 0 & -1 & 0 & 0 & -4 & 0 & 0 \\
0 & 0 & 0 & 0 & -1 & 6 & 0 & 3 & 8 & 0 & 2 & 4 \\
0 & 0 & 0 & -2 & 0 & 0 & 6 & -2 & 0 & 0 & -2 & -4 \\
0 & 0 & 0 & 0 & 0 & -\frac{1}{2} & -1 & \frac{1}{4} & 0 & -2 & 0 & 0 \\
0 & 0 & -1 & 0 & -1 & 0 & 0 & -3 & -16 & 0 & 0 & 0 \\
0 & 0 & 0 & -2 & -\frac{1}{2} & 0 & 0 & 1 & 4 & 0 & 0 & 0 \\
0 & 0 & 0 & 0 & 0 & 0 & 0 & 0 & 0 & -6 & -\frac{1}{2} & -1
\end{array}\right) .
$$

The null space of this matrix (which has dimension 6) can be written as direct sum of the 5 counterterms:

$$
\begin{aligned}
& \sigma D^{2} R, \quad \sigma D^{2}(\Omega-2 \chi), \quad \sigma\left(12 \chi^{2}-4 \chi \Omega-\frac{1}{2} \Omega_{A B} w^{A} w^{B}\right), \\
& \sigma\left(2 R \chi-2 R \Omega+\frac{w^{A} D_{A} R}{2}-6 D^{2} \chi\right), \\
& \sigma\left(-9 \chi^{2}-\Omega^{2}+6 \chi \Omega+\Omega_{A B}^{2}+\frac{\chi R}{2}\right),
\end{aligned}
$$

and the remaining type B anomaly $J^{2}$.

\section{Acknowledgments}

We are grateful to Shira Chapman and Igal Arav for useful discussions. 
Open Access. This article is distributed under the terms of the Creative Commons Attribution License (CC-BY 4.0), which permits any use, distribution and reproduction in any medium, provided the original author(s) and source are credited.

\section{References}

[1] I. Arav, S. Chapman and Y. Oz, Non-relativistic scale anomalies, arXiv:1601.06795 [INSPIRE]. 